\title{
Benefits and Use of Blockchain Technology to Human Resources Management: A Critical Review
}

\author{
Candy So Suk Yi (Candice), \\ MSc in International Management with HRM, \\ University of Roehampton (UK) \& MBA, University of South Australia (Australia)
}

Eric Yung,

JSpectrum Software Pte Ltd., Singapore

Dr. Christopher Fong, Singapore

Shilpi Tripathi, Singapore

Received: March 15, 2020 Accepted: April 15, 2020 Online published: April 29, 2020

doi:10.5296/ijhrs.v10i2.16932ＵRL: https://doi.org/10.5296/ijhrs.v10i2.16932

\begin{abstract}
Globalization brings advantages to worldwide awareness and cross-border technology dissemination in two respects to enable nations to readily access foreign expertise and enhance international competition from the increase of emerging market companies, as well as innovation and the introduction of foreign innovations (Aslam et al., 2018). Human Resources (HR) nowadays generally faces various difficulties in the world internet era and spends a lot of time connecting, screening, and verifying the resume of applicants, conducting credentials verifications, and checking backgrounds to reduce the likelihood of poor recruitment. For example, recruiters connect the profile of candidates from different channels such as direct application, recruitment agency, and social media; and hiring resume verifications is therefore a bottleneck. Hong Kong's telecommunications industry is totally privately-owned and faces no restriction on foreign investment, and it is also open for competition. Use of blockchain in the twenty-first for the period from 2004 to 2014, an instance of international expertise and technology will increase innovation ability and labour productivity development. Experts say that obtaining verification of credentials using blockchain can reduce costs and delays, increase confidence and increase hiring automation
\end{abstract}


(Han, 2017). Background checks on shortlisted candidates / applicants' lies are used to find increasing numbers of companies on their profiles to get job opportunities (Wood et al., 2007 cited in Brody, Richard G, 2010).

Keywords: blockchain, blockchain technology, human resources, human resources management, fraud preventions, cyber security, background check, reference check, recruitment, hiring, on-boarding and off-boarding

\section{Introduction}

\subsection{Background to the Study}

Globalization brings advantages to worldwide awareness and cross-border technology dissemination in two respects to enable nations to readily access foreign expertise and enhance international competition from the increase of emerging market companies, as well as innovation and the introduction of foreign innovations (Aslam et al., 2018). Human Resources (HR) nowadays generally faces various difficulties in the world internet era and spends a lot of time of connecting, screening, and verifying the resume of applicants, conducting credentials verifications, and checking backgrounds to reduce the likelihood of poor recruitment. For example, recruiters connect the profile of candidates from different channels such as direct application, recruitment agency, and social media; and hiring resume verifications is therefore a bottleneck. Use of blockchain in the twenty-first for the period from 2004 to 2014, an instance of international expertise and technology will increase innovation ability and labour productivity development. Experts say that obtaining verification of credentials using blockchain can reduce costs and delays, increase confidence and increase hiring automation (Han, 2017). Background checks on shortlisted candidates / applicants' lies are used to find increasing numbers of companies on their profiles to get job opportunities (Wood et al., 2007 cited in Brody, Richard G, 2010).

Hall (2017) shares a survey by CareerBuilder about the cost of bad hire, the findings are 37 percent of employers cited less productivity, 32 percent wasted time to recruit and trainer employees, and 31 percent found unmatched and poor work quality; and the result was 50 percent of new hirers quitted within six months. The cost of bad hiring reduces productivity, cultural imbalance, disrupt reputation and financial impact to organization (Girdler, 2018). The cyber-attack brings the conflicts of stealing information of a person or organization, an example of Huawei 5G. (Kahn, 2018). Hong Kong's telecommunications industry is totally privately-owned and faces no restriction on foreign investment, and it is also open for competition. The leading telecom operators are Hong Kong Telecom (HKT), Hong Kong Broadband Network and Hutchison Telecoms Hong Kong that are fully private-owned.

The pool of telecom candidates is narrow and extended to non-local candidates whose background in knowledge and skills related to telecom or mobile network such as 5G. Recruiters generally perform reference checks (mostly job referencing) and or hire third-party agencies for background checks to minimize the likelihood of poor hiring. Background check is a common check that includes schooling and vocational training, job history, credit, criminal, drug trafficking, driving records, health checks, etc. 
Using blockchain technology in human resources may also be the necessary instrument for speeding up the time, resources and price of job information on credential verifications. Further research on the effect of blockchain or blockchain technology in Hong Kong's telecommunications industry's recruitment and offboarding; and the future of blockchain is about how to provide multi-channel recruitment and exit methods to blockchain technology.

\subsection{Statement of Blockchain Platform}

Satoshi Nakamoto, a Japanese who created blockchain in 2008, and the underlying data structure under Bitcoin (Nakamoto, 2008). Blockchain, is a distributed database which can be de-centralized, traceable, non-tampering, secure, reliable, integrate, and peer-to-peer protocol, digital encryption technology (Wu \& Li, 2018 cited in Sakho et al., 2019). The distributed database in blockchain acts as "ledger" to record transactions between two parties in a verifiable and permanent way (Iansiti and Lakhani, 2017). Blockchain provides irreversibility of records, once a transaction is entered in the database and the records cannot be changed. (Murck, 2017) Blockchain platform can be divided into alliance chain of public and private (Kosha et al., 2016 cited in Sakho et al., 2019). The nodes in public chain can be easily joined or exited but private chain is only restricted to participating nodes to access and also limit to number of participating agencies (Sakho et al., 2019). According to Melanie Swan, founder of the Blockchain Science Institute, blockchain technology can be undergone of three phrases of 1.0 for currencies, 2.0 for contracts and 3.0 for science, health, government, literacy, culture and art (Swan, 2015 cited in Sakho et al., 2019). Thus, blockchain 3.0 is being popular use to non-finance but across all functions, business and industries. A blockchain is an increasing list of documents, called blocks, which are connected using cryptography. Each block includes the earlier block's cryptographic hash, timestamp, and information on transactions. A blockchain is resistant to changes in the information by design and can be used in a variety of different applications and disciplines of businesses.

Political and economic factors are more financially related among four drivers, while social and technical aspects can be applied to all functions, businesses and industries. More blockchain technology enhancements are really a matter of solving the issues. Industries 5.0, an intelligent hiring of employees and a management system across all industries are needed (Onik et al., 2018). Tapscott and Tapscott (2016) shares the current tool mostly is centralized to create a problem of "trust and capability", but blockchain-based system allows employees and companies to keep data rather than relying on social network companies. An example of employers who use internal Human Resources Information System (HRIS) (either self-developed or third-party platform) to create, access, edit and change employees' data. The information is not precise and checked, and therefore the information has no significance on the departure of the staff.

The potential use of blockchain is to check and evaluate candidates' credential preparation, abilities and efficiency, and their contribution to HR tasks (PwC, 2017). In Hong Kong, a lady who used fake credentials to get $\mathrm{HK} \$ 88,000$ a month, a CEO was accused of fraud, an example of fake resume (SCMP, 2016). Another example of fake certificates was that of 11,000 government jobs in Mumbai, India (The Times of India, 2019). HR Professionals are 
usually concerned with data privacy and security, and also time, cost and resources effectiveness using technology to manage the workflow. The evolving landscape of the workforce, such as outsourcing, contracting, temporary employment, freelancing, and gig economy, brings recruiting difficulties. Some references to employment are difficult to trace and verify. Blockchain technology allows legal work contracts between employer and employee to be protected in accordance with local legalizations (Pinna and Ibba, 2018), An example of smart contracts can be created, checked and traced under legal parties and compliance. The laid off programs at Starhub (laid off 300 employees and restructuring cost of SG $\$ 25$ million) and Optus (laid off 400 or more employees); and also, IT outsourcing and automation trend shifts technical jobs to offshore. A report by Existek (2019) in the leading four countries such as India, China, Malaysia and Indonesia, it is crucial to overcome barriers of language and accurate information by credentials verification and background check.

\subsection{Blockchain Technology Benefits to Human Resources}

There is also a large body of work in this area considering that human resources' function, along with globalization, has continuously risen, altering company climate, organizational structure, and innovations in culture and technology. HR practitioners play a main role in providing consultancy to the organisation as a "change transformation agent," and members of its leadership make actual behavioural changes influencing organisational efficiency (Deshler, 2016). According to Andrew Spencer, a UK based HR technology consultant/researcher who claims that the four areas where blockchain can be used are credential verification, worker payments, work matching platforms, and identity management (Ahmed, 2019 cited in People Matters, 2019). Blockchain technology is one of the safe technologies that provides HR with a platform for streamlining working procedures and more time for addressing issues and communicating with stakeholders. Blockchain can recognize prospective hazards and procedures of inefficiency in activities requiring resources such as manpower, time and money can be handled as suitable for transforming blockchain technology to decrease information collection and verification. An instance is using a blockchain ledger since it is a reliable instrument for verifying the history of applicants (Aishwarya, 2018). Blockchain technology can help in hiring to save cost of communication and logistics for the purpose of verification - An example of saving cost of communication and logistics is employers with a ready reference and verifiable $\mathrm{CV}$ of the candidates (Hegadekatti, 2018).

\subsection{Blockchain Technology Add-Value to Hiring and Off-Boarding}

A number of studies has examined the recruitment is the process of searching the right person who may or may not be interested to change their current job to fill the job opportunity for an organisation (Phillips and Gully, 2012 cited in Koch et al., 2018). Hiring cycle includes connecting, screening, and interviewing applicants, offering preparation, background checking, on-boarding, and off-boarding, spending a lot of time, cost, and manpower processing verifications and administrative work. A study of 344,250 interviews in six Glassdoor demonstrates that recruitment is a time-consuming method in which work interviews are prolonging (3.3 days to 3.7 days since 2009), complex interviews and 
background checks for employees (Chamberlain, 2015). The Australian Financial Review described that in 2015 the average time taken to fill a vacant position in Australia was 68 days, with a 50 per cent increase from 2010 (Morshed Chowdhury, 2018). The main challenge, therefore, is how to guarantee accurate and trustworthy data is supplied by applicants. Recruiters generally rely on the resume and/or supporting paperwork submitted by applicants in the hiring process, such as certifications, job reference letters, payslips, tax and insurance evidence. The prevalent practice of verifications is generally handled by inner administrative human resources and/or assigned to third parties and applicants provide contact information (Morshed Chowdhury et al., 2018). Candidates usually provide favourable reference check contact to proceed reference check. Generally, it is not possible to locate in the resume of the applicants for those non-disclosed or negative information. Blockchain technology enables organizations to check candidate authenticity through distributed third-party ledger or through candidate access (key or token). Candidates can create, monitor and maintain access to a blockchain-based record of their certificates of education and training and work history through distributed ledger (Aishwarya, 2018). The potential risks and insufficient protection of privacy, discovery and illegal use of permanent data based on social networking and uncontrollable multiple identities. Another critical issue with blockchain is that digital records are hard to verify (Tucker and Catalini, 2018)

\section{Data Privacy and Security Are Issues in Blockchain Technology}

There is a vast literature on methods to solve this issue that the potential risks and insufficient protection of privacy, discovery and illegal use of permanent data based on social networking and uncontrollable multiple identities. Another critical issue with blockchain is that digital records are hard to verify (Tucker and Catalini, 2018). There is a vast literature on methods to solve this issue using consensus algorithms such as job evidence, stake proof, practical Byzantine fault tolerance, delegated stake proof and scalable Byzantine consensus protocol design and blockchain systems that may not be adequate for future data protection. Ryan Henry and his peers (2019) are discussing another problem of privacy as the present tor limits and may not be sufficient in the future to provide the same amount of safety privacy. B

Besides, Blockchain may not easy to apply globally due to local legislations. For example, Hong Kong has the Rehabilitation of Offenders Ordinance (the ROO) and the Personal Data (Privacy) Ordinance. Employer must obtain candidates' declaration before proceeding official background check.

\subsection{Resume Verification}

A number of studies have examined in Society for Human Resources Management (SHRM) 2016 report that social media platforms allow people to create and upload their own profile without verifications. A finding states that it was 84 percent growth in recruitment via social media, 82 percent of passive job applicants be the top reason that companies use social media for recruitment. Another study by Robert Walters states that the top three social media recruiting platforms for job applicants and employers are LinkedIn, Facebook, and Twitter respectively (Robert Walters Whitepaper). Recruiters connect applicants (active, semi-passive or passive) through social platforms that are not verified. Before posting online, active 
applicants can polish their resume and then apply for a job to the business or recruitment agency. For instance, without verification, an organization can obtain duplicate job applicants from various channels. A study of approximately 5,000 resumes undertaken by the Risk Advisory Group concluded that $80 \%$ of the resumes of applicants found discrepancies with $57 \%$ in academic background and $12 \%$ of the resumes of applicants found falsified in grading (Batzavalis et al., 2018)

The deployment of distributed ledger in academic organizations can help recruiters to verify skills for education. The data can be verified by distributed ledgers such as person (job applicant), government or private (professional universities / bodies / institutes) or government or private third party (background check company / BCHRIS / BCRMS). Some ledgers can even screen applications for unqualified jobs and provide candidates with ranking. Blockchain benefits recruiters with pre-verifications when reaching applicants through social networking to decrease "time, effort and price," speed up the process and job matching. A report states that blockchain can enhance fraud prevention, cybersecurity and data protection in HR (PwC, 2017).

\subsection{Background Checks}

Recruiters face difficulties in verifying the resume of applicants for worldwide recruitment or particular legal blinding. Employing third parties, i.e. background check officer, is a prevalent practice to do checking to promote the recognized candidate's conditional job offer. A written background check approval must have the approval of applicants. Society for Human Resources Management (SHRM) surveys indicate that organizations frequently use background checks. Background checks have been treated as a standard and accepted employment screening; and any candidates who refuses to proceed the check may be treated as un-successful candidates (Brody, 2010). Therefore, blockchain represents an opportunity for third parties, such as employers, to independently and privately verify that shared records (Grech and Camilleri, 2017). Recruiters can access trusted ledger to verify the background of applicants in the history of education and work. Other legal-related information such as credit and criminal records; or private documents such as health records and driving records may still respond to third parties. The candidate's conditional offer requires that the background check has a positive result, otherwise it is disqualified.

\subsection{Employment Records Management}

The issue explored by using HR petitioner spends time of creating and updating the work history of staff and HRIS enrolments. For instance, account formation and termination, enrolment advantages, training, performance review, and separation, and or notification to legal parties such as tax or immigration. Recruiters can verify the candidate's job data via a distributed individual or public / private ledger and enter new employee information into the distributed ledger or BCHRIS for administration purposes such as reference check, pay benefits administration (i.e. health care and medical insurance, and or compensations), and legal applications (tax and or insurance). Blockchain can save time in a repeating data input and administration process and using ledgers to manage employment history rather than conducting reference checks. For example, organizations can create their own blockchain 
using smart contracts for routine operating tasks such as verifying employees' performance, conducting background check and managing information across multiple jobs, managers, and companies (Aishwarya, 2018).

Recruiters / Payroll Administrators provide the identified candidate with an offer package that is subject to his / her last offer. In order to benchmark the new offer package, they can refer to the individual or public / private ledger to trace the proof of employment history such as last position, wage, bonus, allowance and benefits. However, verifications of legal data such as loan status, criminal history, and or health records may not be included in the ledger but may depend on third parties (or the distributed ledger-public or owned by third parties) to process the verification. A candidate's oral or written consent before continuing with inspections. Recruiters prepare employment contracts for candidates to sign before or on the date of boarding. Blockchain offers a trustable ledger - public or private parties to access candidates' background and also speed-up the hiring process.

\section{Conclusions, Future Trends and Implications of Blockchain}

This is generally accepted in the literature from which we can draw the conclusion that HR practitioners completes the process of off-boarding such as payroll, exit interview and update job records. Blockchain can store employment documents in the ledger and parties can access the data subject to the access connections, so there is no need for recruiters, and or applicants (i.e. authorizing parties) to spend time on checking references. A review of major studies in this area confirmed that Blockchain technology benefits HR hiring and off-boarding when it comes to verification and history of candidates' qualifications and also fraud prevention. Blockchain transforms a company into a more structured and well-managed company that enables a hybrid strategy to safe legacy infrastructure and distributed ledger models. Processes involving human resources such as payroll, recruitment and choice, on-boarding and off-boarding waste a lot of time, resources and money and even by third parties. Blockchain searching would be more efficient compared to internet search and would benefit the recruitment process. Blockchains give privacy and screening method for non-bias users. Blockchain development is a real-time application followed by verification, dynamic update and system learning (Onik et al., 2018). Future research should therefore seek to address this issue by more papers talk about technical and cryptographic challenges, more research focus on market design, trust and privacy, and the adoption respective non-adoption of the new technology.

\section{References}

Ahmed, M. (2019). Blockchain can be transformational for future of work: Andrew Spence. People Matters.

https://www.peoplemattersglobal.com/article/techhr-2019/blockchain-can-be-transformationa 1-for-future-of-work-andrew-spence-20994

Aishwarya, N. (2018). Potential impact of blockchain on HR and people management. JETIR September 2018, 5(9), 127-130. http://www.jetir.org/papers/JETIRA006284.pdf 
Aslam et al., (2018). Globalization Helps Spread Knowledge and Technology Across Borders. IMFBlog.https://blogs.imf.org/2018/04/09/globalization-helps-spread-knowledge-and-techno logy-across-borders/

Batzavalis et al., (2018). A Platform for Leveraging Blockchain Technology for the Storage. Issuance and Authentication of Academic Credentials. TrustED, 1-55. https://ico.trusteducation.io/wp-content/uploads/2018/11/TrustED-Whitepaper.pdf

Brody, R. G. (2010). Beyond the Basic Background Check: Hiring the "Right" Employees. Management Research Review, 33(3), 210-223. https://doi.org/10.1108/01409171011030372

Chamberlain, A. (2015). Why Is Hiring Taking Longer? New Insights from Glassdoor-data https://www.glassdoor.com/research/app/uploads/sites/2/2015/06/GD_Report_3.pdf

Christidis, K., \& Devetsikiotis, M. (2016) Blockchains and Smart Contracts for Internet of Technology. IEEE, 4, 2292-2303. https://doi.org/10.1109/ACCESS.2016.2566339

Deshler, R. (2016). HR's role as change transformation agent. Strategic HR Review. https://doi.org/10.1108/SHR-03-2016-0028

Girdler, S. (2018). The Real Impact Of A Bad Hire For Your Business. https://www.entrepreneur.com/article/313729

Grech, A., \& Camilleri, A. F. (2017). Blockchain in education.

Hall, B. (2017). The Cost of a Bad Hire. Human Resources Online. Available at: https://www.humanresourcesonline.net/the-cost-of-a-bad-hire/

Han, Y. J. (2017). Blockchain-Verified Credentials Could Change the Game of Hiring. Available at: https://news.bloomberglaw.com/daily-labor-report/blockchain-verified-credentials-could-cha nge-the-game-of-hiring?context=article-related

Hegadekatti, K. (2018). Blockchain and Human Resources Management. Available at SSRN 3232203. https://doi.org/10.2139/ssrn.3232203

Iansiti, M., \& Lakhani, K. A. (2017). The Truth About Blockchain. Harvard Business Review. https://hbr.org/2017/01/the-truth-about-blockchain

Johnson, R. D., \& Guetta, H. G. (2011). Transforming HR Through Technology. SHRM.https://www.shrm.org/hr-today/trends-and-forecasting/special-reports-and-expert-vie ws/Documents/HR-Technology.pdf

Kahn, A. R. (2018) Economic Espionage in 2017 and Beyond: 10 Shocking Ways They Are Stealing Your Intellectual Property and Corporate Mojo; https://mail.google.com/mail/u/0/\#inbox

Koch, et al., (2018). The impact of social media on recruitment: Are you LinkedIn? SA Journal of Human Resource Management (1-14). https://doi.org/10.4102/sajhrm.v16i0.861 


\section{$\triangle 1$ Macrothink}

International Journal of Human Resource Studies

ISSN 2162-3058

Morshed, C. et al., (2018) Blockchain for Fraud Prevention: A Work-History Fraud Prevention System. Conference: 2018 17th IEEE International Conference on Trust, Security And Privacy In Computing And Communications/ 12th IEEE International Conference On Big Data Science And Engineering (TrustCom/BigDataSE). https://www.researchgate.net/publication/327484134_Blockchain_for_Fraud_Prevention_A_ Work-History_Fraud_Prevention_System

Murck, P. (2017). Who controls the blockchain? Harvard Business Review. https://hbr.org/2017/04/who-controls-the-blockchain

Nakamoto, S. (2008). Bitcoin: A Peer-to-Peer Electronic Cash System. pp. 1-9. https://bitcoin.org/bitcoin.pdf

Nofer et al., (2017) Blockchain. Business Information System Engineering, 59(3), 183-187. https://doi.org/10.1007/s12599-017-0467-3

Onik, et al., (2018) A Recruitment and Human Resource Management Technique Using Blockchain Technology for Industry 4.0, 11-16 IET. https://doi.org/10.1049/cp.2018.1371

Pinna, A., \& Ibba, S. (2018) A Blockchain-based decentralized system for proper handling of temporary employment contracts. SAI 2018. Intelligent Computing, 1231-1243. https://link.springer.com/chapter/10.1007\%2F978-3-030-01177-2_88

PwC. (2017) How Blockchain Technology Could Impact HR and the World of Work. https://www.pwc.co.uk/issues/futuretax/assets/blockchain-can\%20impact-hr.pdf

Ryan, H., Amir, H., \& Aniket, K. (2019). "Blockchain Access Privacy: Challenges and Directions"

https://www.cs.purdue.edu/homes/akate/publications/BlockchainAccessPrivacy_preprint.pdf

Sakho, et al., (2019). Privacy Protection Issues in Blockchain Technology. International Journal of Computer Science and Information Security (IJCSIS), 17(2), 124. https://www.academia.edu/38529469/Privacy_Protection_Issues_in_Blockchain_Technology

Tapscott, \& Tapscott. (2016). The Impact of the Blockchain Goes Beyond Financial Services. Harvard

Business

Review.

https://hbr.org/2016/05/the-impact-of-the-blockchain-goes-beyond-financial-services

Tucker, C., \& Catalini, C. (2018). What Blockchain Can’t Do? Harvard Business Review. https://hbr.org/2018/06/what-blockchain-cant-do

Woodside et al., (2017) Blockchain Technology Adoption Status and Strategies. Journal of International Technology and Information Management, 26(32). https://scholarworks.lib.csusb.edu/cgi/viewcontent.cgi?referer=https://www.google.com/\&htt psredir $=1 \&$ article $=1300 \&$ context $=$ jitim 


\section{Copyright Disclaimer}

Copyright for this article is retained by the author(s), with first publication rights granted to the journal.

This is an open-access article distributed under the terms and conditions of the Creative Commons Attribution license (http://creativecommons.org/licenses/by/4.0/). 